\title{
Discovery of Scattering Polarization in the Hydrogen Ly $\alpha$ Line of the Solar Disk Radiation
}

\author{
R. Kano ${ }^{1}$, J. Trujillo Bueno ${ }^{2,3,4}$, A. Winebarger ${ }^{5}$, F. Auchère $^{6}$, N. Narukage ${ }^{1}$, R. Ishikawa ${ }^{1}$, K. Kobayashi ${ }^{5}$, T. Bando $^{1}$, \\ Y. Katsukawa ${ }^{1}$, M. Kubo ${ }^{1}$, S. Ishikawa ${ }^{7}$, G. Giono ${ }^{1,8}$, H. Hara ${ }^{1}$, Y. Suematsu ${ }^{1}$, T. Shimizu ${ }^{7}$, T. Sakao ${ }^{7}$, S. Tsuneta ${ }^{7}$, K. Ichimoto ${ }^{9}$, \\ M. Goto ${ }^{10}$, L. Belluzzi ${ }^{11,12}$, J. Štěpán ${ }^{13}$, A. Asensio Ramos ${ }^{3}$, R. Manso Sainz ${ }^{14}$, P. Champey ${ }^{15}$, J. Cirtain ${ }^{16}$, B. De Pontieu ${ }^{17}$, \\ R. Casini ${ }^{18}$, and M. Carlsson ${ }^{19}$ \\ ${ }^{1}$ National Astronomical Observatory of Japan, 2-21-1 Osawa, Mitaka, Tokyo 181-8588, Japan; ryouhei.kano@nao.ac.jp \\ ${ }^{2}$ Instituto de Astrofísica de Canarias, La Laguna, Tenerife, E-38205, Spain \\ ${ }^{3}$ Departamento de Astrofísica, Universidad de La Laguna, E-38206 La Laguna, Tenerife, Spain \\ ${ }^{4}$ Consejo Superior de Investigaciones Científicas, Spain \\ ${ }^{5}$ Marshall Space Flight Center, National Aeronautics and Space Administration (NASA), Huntsville, AL 35812, USA \\ ${ }^{6}$ Institut d'Astrophysique Spatiale, Université Paris Sud, Batiment 121, F-91405 Orsay, France \\ ${ }^{7}$ Institute of Space and Astronautical Science, Japan Aerospace Exploration Agency, 3-1-1 Yoshinodai, Chuo, Sagamihara, Kanagawa 252-5210, Japan \\ ${ }^{8}$ The Graduate University for Advanced Studies (Sokendai), Hayama, Kanagawa 240-0193, Japan \\ ${ }^{9}$ Hida Observatory, Kyoto University, Takayama, Gifu 506-1314, Japan \\ ${ }^{10}$ National Institute for Fusion Science, Toki, Gifu 509-5292, Japan \\ ${ }^{11}$ Istituto Ricerche Solari Locarno, CH-6605 Locarno Monti, Switzerland \\ ${ }^{12}$ Kiepenheuer-Institut für Sonnenphysik, D-79104 Freiburg, Germany \\ ${ }^{13}$ Astronomical Institute, Academy of Sciences of the Czech Republic, 25165 Ondrejov, Czech Republic \\ ${ }^{14}$ Max-Planck-Institut für Sonnensystemforschung, Justus-von-Liebig-Weg 3, D-37077 Göttingen, Germany \\ ${ }^{15}$ University of Alabama in Huntsville, 301 Sparkman Drive, Huntsville, AL 35899, USA \\ ${ }^{16}$ University of Virginia, Department of Astronomy, 530 McCormick Road, Charlottesville, VA 22904, USA \\ ${ }^{17}$ Lockheed Martin Solar \& Astrophysics Laboratory, Palo Alto, CA 94304, USA \\ ${ }^{18}$ High Altitude Observatory, National Center for Atmospheric Research, Post Office Box 3000, Boulder, CO 80307-3000, USA \\ ${ }^{19}$ University of Oslo, Postboks 1029 Blindern, NO-0315 Oslo, Norway \\ Received 2017 March 24; accepted 2017 March 26; published 2017 April 10
}

\begin{abstract}
There is a thin transition region (TR) in the solar atmosphere where the temperature rises from $10,000 \mathrm{~K}$ in the chromosphere to millions of degrees in the corona. Little is known about the mechanisms that dominate this enigmatic region other than the magnetic field plays a key role. The magnetism of the TR can only be detected by polarimetric measurements of a few ultraviolet (UV) spectral lines, the Ly $\alpha$ line of neutral hydrogen at $121.6 \mathrm{~nm}$ (the strongest line of the solar UV spectrum) being of particular interest given its sensitivity to the Hanle effect (the magnetic-field-induced modification of the scattering line polarization). We report the discovery of linear polarization produced by scattering processes in the Ly $\alpha$ line, obtained with the Chromospheric Lyman-Alpha SpectroPolarimeter (CLASP) rocket experiment. The Stokes profiles observed by CLASP in quiet regions of the solar disk show that the $Q / I$ and $U / I$ linear polarization signals are of the order of $0.1 \%$ in the line core and up to a few percent in the nearby wings, and that both have conspicuous spatial variations with scales of $\sim 10$ arcsec. These observations help constrain theoretical models of the chromosphere-corona TR and extrapolations of the magnetic field from photospheric magnetograms. In fact, the observed spatial variation from disk to limb of polarization at the line core and wings already challenge the predictions from three-dimensional magnetohydrodynamical models of the upper solar chromosphere.
\end{abstract}

Key words: magnetic fields - polarization - Sun: chromosphere - Sun: transition region - Sun: UV radiation

\section{Introduction}

The upper layers of the solar chromosphere, and the transition region (TR) in particular, are key to understanding several of the most fundamental problems in astrophysics such as the source of the solar wind, the energization of the corona, or the acceleration of energetic particles (e.g., Priest 2014). Most of what we know about this puzzling atmospheric region has been learned from ground-based observations in the $\mathrm{H} \alpha$ line (e.g., Kneer 2010; Rutten \& Rouppe van der Voort 2017) and by combining space-based observations of the radiation intensity in ultraviolet (UV) spectral lines and magnetohydrodynamical numerical simulations (e.g., Hansteen et al. 2014; Pereira et al. 2014). Yet, we lack quantitative information on the single most important physical parameter: its magnetic field. To explore the magnetic field of the upper solar chromosphere and TR requires spectropolarimetry, i.e., the ability to detect the polarization state of photons in magnetically sensitive spectral lines, at UV wavelengths where photons are relatively scarce. In particular, the magnetic field of the rarified plasma of the chromosphere-corona TR can be probed only through a handful of UV spectral lines sensitive to the Hanle effect (the magnetic-field-induced modification of the line scattering polarization; see Landi Degl'Innocenti \& Landolfi 2004), but their observation and modeling are not easy (e.g., Trujillo Bueno 2014). On the one hand, these wavelengths cannot be observed from the ground, which poses a clear instrumental and observational challenge; on the other, the formation of the polarization profiles of these lines involves complex atomic and radiative mechanisms whose impact is only known theoretically, since the physical conditions of the TR are not accessible in terrestrial laboratories. These difficulties have left the strength and geometry of the magnetic 
field in the numerical models of the upper solar chromosphere largely unconstrained. Unable to directly confront models to observable quantities sensitive to the magnetic field, complex extrapolations of solar surface magnetograms are instead used, which are problematic because they are difficult or impossible to validate against actual observations (e.g., De Rosa et al. 2009).

The dominant spectral feature in the UV solar spectrum is the hydrogen Ly $\alpha$ emission line at $121.6 \mathrm{~nm}$, which forms all through the upper chromosphere and in the TR. This resonance line is dominated by scattering, and hence it could show a linear polarization pattern sensitive to the geometry of the formation region and to the presence of magnetic fields through the Hanle effect. A first-and for almost 40 years, onlyattempt to detect solar limb polarization in this line using a slitless spectropolarimeter failed, presumably due to in-flight degradation of its efficiency (Stenflo et al. 1980). Recent theoretical work has predicted that in models of the solar atmosphere, with magnetic fields between 10 and 100 Gauss, the measurable level of linear polarization in the Ly $\alpha$ radiation of the solar disk should be of the order of $0.1 \%$ in the line core and up to a few percent in the nearby wings (Trujillo Bueno et al. 2011; Belluzzi et al. 2012; Štěpán et al. 2015).

In order to achieve the first measurement of the linear polarization produced by scattering processes in the hydrogen Ly $\alpha$ line radiation of the solar disk, we have developed the Chromospheric Lyman-Alpha Spectro-Polarimeter (CLASP; Kano et al. 2012; Kobayashi et al. 2012). CLASP was successfully launched from White Sands Missile Range by a NASA sounding rocket on 2015 September 3, and the aim of this Letter is to show and discuss the results of these unprecedented spectropolarimetric observations.

\section{The Instrument}

The CLASP (Narukage et al. 2015, 2017) consists of a Cassegrain telescope with an aperture of $27 \mathrm{~cm}$; a spectropolarimeter to measure the wavelength variation of the Stokes $I, Q / I$, and $U / I$ profiles along the 400 arcsec covered by the spectrograph's slit (nearly half the solar radius); and a slit-jaw optical system to take fast-cadence $(0.6 \mathrm{~s})$ chromospheric images with a Ly $\alpha$ broadband filter (FWHM $=7 \mathrm{~nm})$. The spectropolarimeter uses a rotating half-waveplate for the Ly $\alpha$ wavelength (Ishikawa et al. 2013, 2015), located in front of the slit, which modulates the polarization of the incident radiation four times per rotation (one rotation takes $4.8 \mathrm{~s}$ ). It also uses a single concave diffraction grating, mounted in the inverse Wadsworth configuration, which serves both as the spectral dispersion element and beam splitter (Narukage et al. 2015). Each of the resulting optically symmetric channels goes through a linear-polarization analyzer mounted $90^{\circ}$ from each other to simultaneously measure two orthogonal polarization states. From the observed signals in both channels, we derive the wavelength variation of the Stokes $I$ profile and of the fractional polarization $(Q / I$ and $U / I)$ applying dual channel demodulation (Ishikawa et al. 2014), with plate scales of $0.0048 \mathrm{~nm} \mathrm{pixel}^{-1}$ and 1.1 arcsec pixel ${ }^{-1}$. Additionally, since the rotating half-waveplate was located in front of the slit and the fold mirror of the slit-jaw system behaved as a partial linear polarizer, the CLASP slit-jaw system itself also provided images of the broadband $Q / I$ fractional polarization, in addition to the intensity images mentioned above.

\section{Observational Results}

During its ballistic flight on 2015 September 3, CLASP observed first a quiet region at the solar disk center during 17:02:53-17:03:09UT in order to quantify the instrumental polarization, and we confirmed that the spurious polarization is one order of magnitude smaller than $0.1 \%$ (Giono et al. 2016, 2017). Afterward, during 17:03:36-17:08:25UT, CLASP observed relatively quiet regions of the Sun (Figure 1 (Aa)), with the slit oriented radially from 20 arcsec off-limb toward the disk center.

Figure 1(B) shows the wavelength variation of Stokes $I, Q / I$, and $U / I$ measured by CLASP at each pixel along the slit. The intensity emission profile shows significant spatial fluctuations on scales of $\sim 10$ arcsec, which essentially corresponds to the chromospheric network that results from intense photospheric magnetic field concentrations and related heating events: bright in the network and dark in the internetwork (Figure 1(Aa); see also Kubo et al. 2016). In the wings, $Q / I$ is negative (the linear polarization is perpendicular to the limb) with amplitudes larger than $1 \%$ and increasing up to $\sim 6 \%$ toward the limb (i.e., with a very clear center-to-limb variation, hereafter CLV). The $U / I$ wing signals are also very significant $(>1 \%)$, with positive and negative values fluctuating around zero on spatial scales of $\sim 10$ arcsec; they are a clear observational signature of the breaking of the axial symmetry (around the solar local vertical) of the Ly $\alpha$ radiation in the solar chromosphere, caused by the horizontal atmospheric inhomogeneities of the solar chromospheric plasma. At the line center, the $Q / I$ and $U / I$ signals on the solar disk are $\sim 0.1 \%$, and fluctuating around zero on spatial scales of $\sim 10$ arcsec. However, at the off-limb positions sampled by the spectrograph's slit the $Q / I$ line-center signals are predominantly positive (parallel to the solar limb) with amplitudes of about $+0.5 \%$. Note that although weak $(\sim 0.1 \%)$, the measured $Q / I$ and $U / I$ line-core signals are an order of magnitude larger than the instrumental polarization level (Ishikawa et al. 2014; Giono et al. 2016, 2017) of CLASP.

The overall shape of the $Q / I$ profiles (Figure 2), as well as the very significant negative $Q / I$ signals in the wings and their clear CLV (Figures 1(B) and 3), are essentially consistent with the theoretical predictions (Belluzzi et al. 2012). The $Q / I$ and $U / I$ line-center amplitudes $(<1 \%)$ also follow the theoretical expectations for moderate $(\sim 3 \mathrm{arcsec})$ spatial resolution observations (Trujillo Bueno et al. 2011; Štěpán et al. 2015), such as those obtained by CLASP. However, the $Q / I$ linecenter signals do not show any clear CLV, in marked contrast with the results of radiative transfer calculations in onedimensional (1D) and three-dimensional (3D) models of the solar atmosphere (Trujillo Bueno et al. 2011; Štěpán et al. 2015).

Spatial fluctuations on scales of $\sim 10$ arcsec are seen both in intensity $(I)$ and linear polarization $(Q / I, U / I)$. Maxima and minima of intensity along the slit in the line core are systematically shifted from those in the wings (Figure 3), the shift becoming more pronounced closer to the limb (especially at slit locations above $100 \mathrm{arcsec}$ ). This effect is due to the different optical depths in the core and wings, and hence to the different geometrical heights they sample (higher in the core, deeper in the wings). Interestingly, maxima of intensity often match minima of total linear polarization $\left(\mathrm{LP}=\sqrt{Q^{2}+U^{2}} / I\right)$ on the Ly $\alpha$ wings and vice versa, as shown by the colored segments in Figure 4(A). The anticorrelation between $I$ and LP is even more noticeable after removing from LP its CLV, proportional to 


\section{A: slit-jaw data}
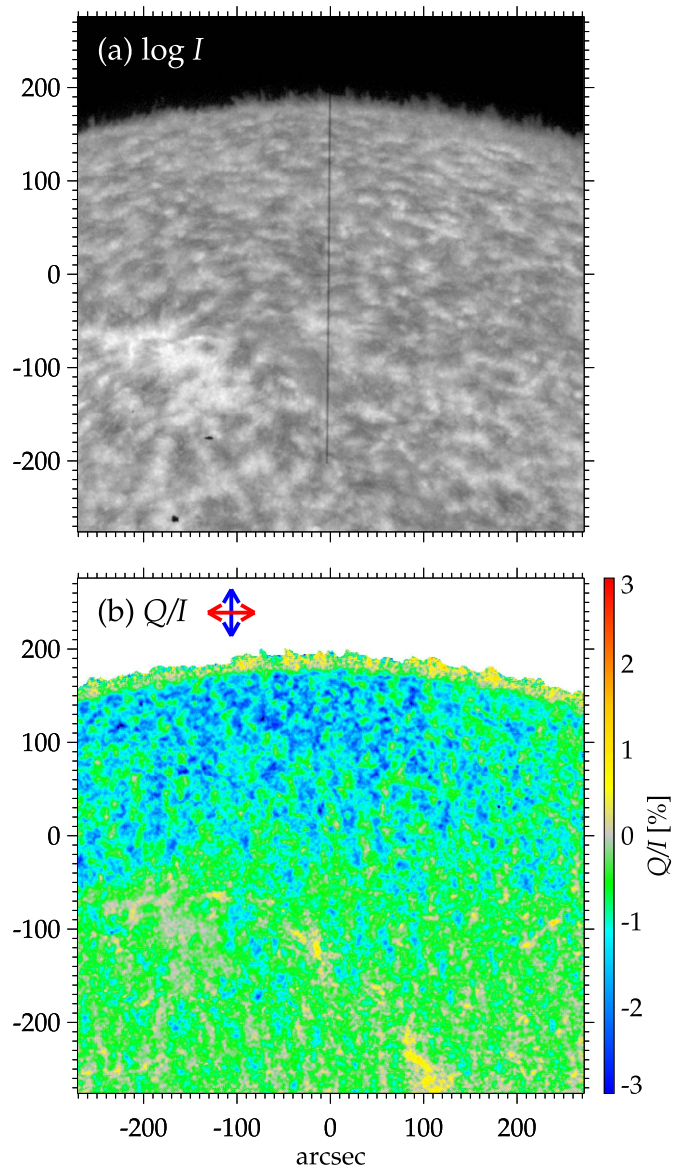

B: spectro-polarimeter data

(a) $\log I$

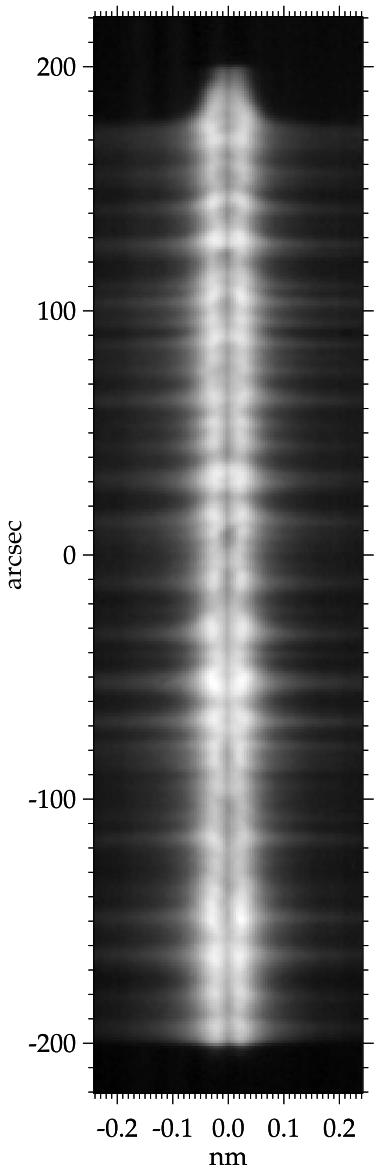

(b) $Q / I$

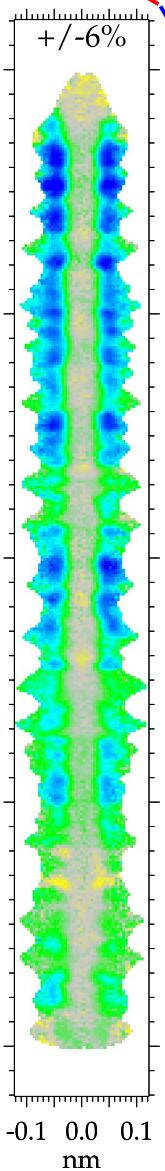

(c) $Q / I$

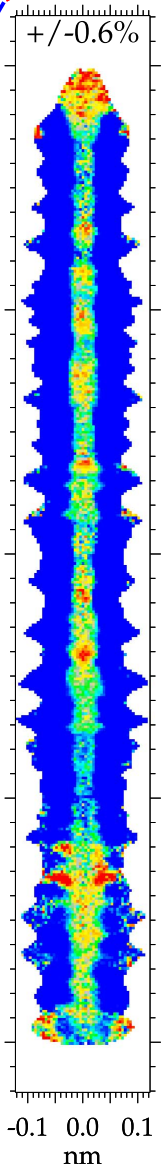

(d) $U / I$

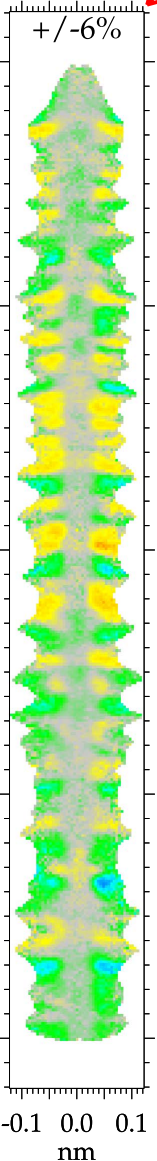

(e) $U / I$

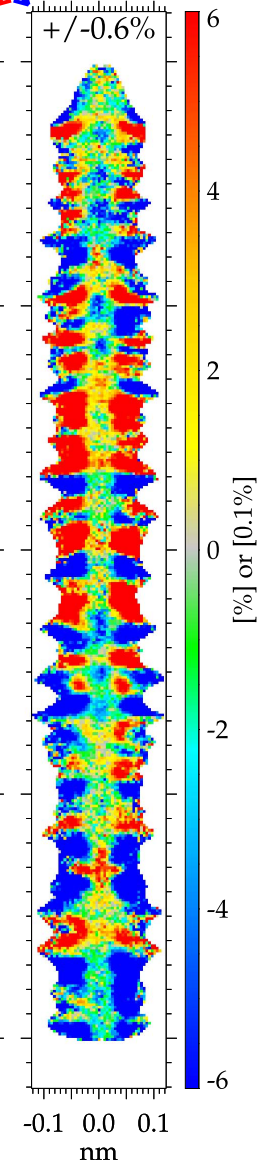

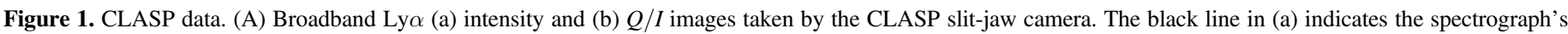

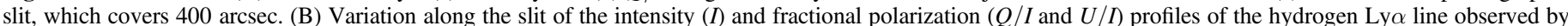

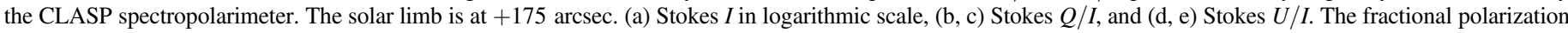

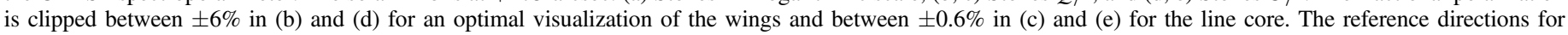
positive Stokes $Q$ and $U$ are indicated by the red arrows in the corresponding (B) panels.
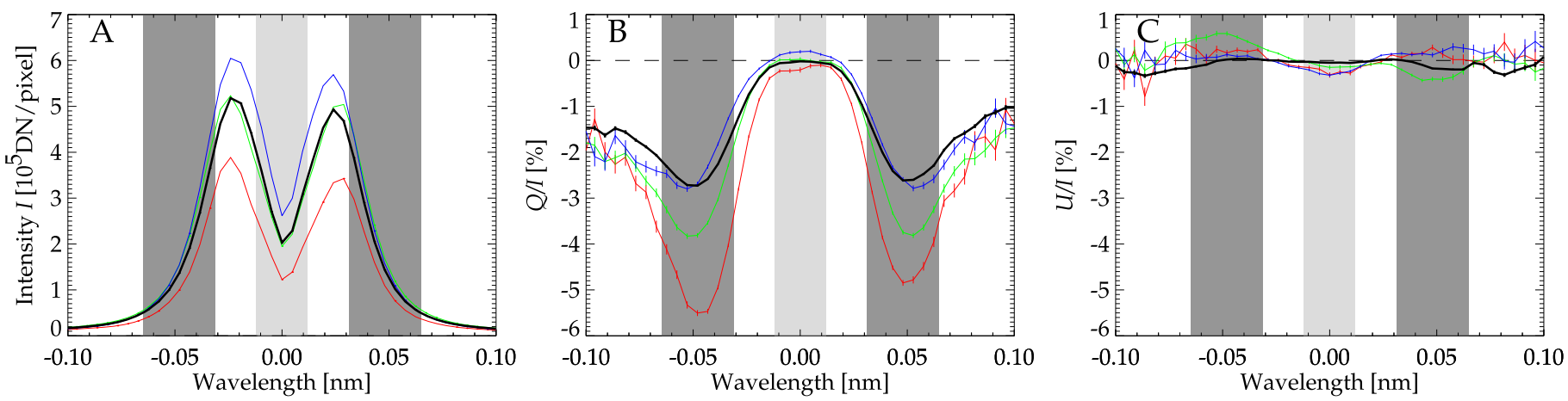

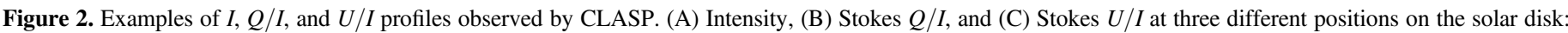

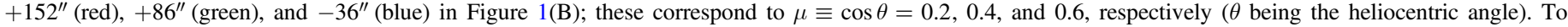

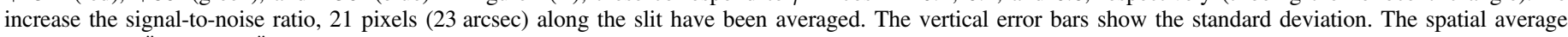
between $-198^{\prime \prime}$ and $+164^{\prime \prime}$ in Figure 1(B) is shown by the black curves.

$\left(1-\mu^{2}\right)$. In some places, even in the Ly $\alpha$ line core, LP is anticorrelated with the intensity (Figure 4(C)). The fact that regions with lower line intensity are, on average, more polarized than the high-intensity regions had been predicted by radiative transfer investigations of the Ly $\alpha$ scattering polarization in 3D models of the solar atmosphere (Štěpán et al. 2015).
The CLASP slit-jaw system also, serendipitously, provided images of the broadband $Q / I$ fractional polarization (Figure 1 $(\mathrm{Ab})$ ), in addition to the intensity images (Figure 1(Aa)). Since the Ly $\alpha Q / I$ amplitudes are much larger in the wings than in the core, the broadband $Q / I$ signal that results from the wavelength-integrated Stokes $I$ and $Q$ profiles is dominated by 

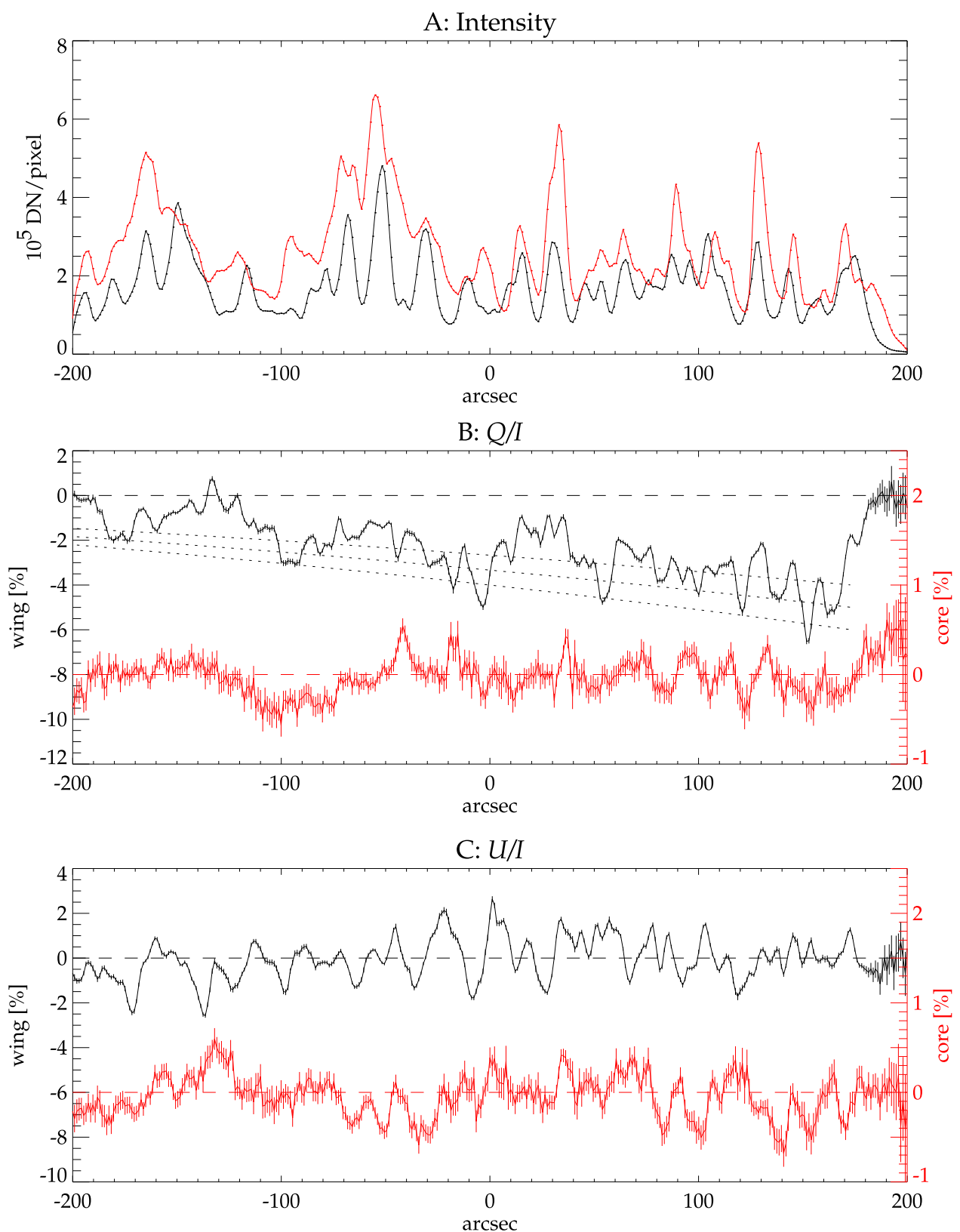

Figure 3. Spatial variations along the slit. (A) Intensity, (B) Stokes $Q / I$, and (C) Stokes $U / I$. The black curves correspond to the Ly $\alpha$ wing intervals given by the dark gray areas of Figure 2, while the red curves correspond to the Ly $\alpha$ core interval given by the light gray area of Figure 2 . The dotted lines in panel (B) indicate the $\left(1-\mu^{2}\right)$ CLV trend.

the polarization in the wings. Interestingly enough, a comparison of this $Q / I$ image with the intensity image clearly shows that the low-intensity regions (e.g., quiet-Sun areas) are indeed more polarized than the high-intensity regions (e.g., plage areas).

\section{Discussion}

In general, there is a good agreement between the theoretical predictions and the spectropolarimetric observations obtained by CLASP. This is far from trivial since the quantum theory of polarization in spectral lines taking into account partial frequency redistribution phenomena is still under development. Many of the subtle spectroscopic and radiative transfer effects it predicts (and explains) take place only in the extremely rarefied but generally optically thick plasma of a stellar atmosphere (e.g.,
Landi Degl'Innocenti 1998; Stenflo et al. 2000; Manso Sainz \& Trujillo Bueno 2003) and cannot be investigated through specifically dedicated laboratory experiments.

The $Q / I$ pattern observed by CLASP, with its strong $(>1 \%)$ near-wing polarization signals, demonstrates that the underlying physical mechanism is the joint action of partial frequency redistribution and $J$-state interference (i.e., in the scattering events the photon goes through the two upper $J$ levels of $\operatorname{Ly} \alpha$ at the same time). Without $J$-state interference, the $Q / I$ wing signals would be significantly weaker (see Belluzzi et al. 2012), contrary to the observations by CLASP.

The complex polarization patterns shown in Figure 1(B) and especially the very significant polarization amplitudes at the Ly $\alpha$ wings are very sensitive to the thermal structure of the upper solar chromosphere, while the line core polarization is sensitive (via the Hanle effect) to the presence of weak 

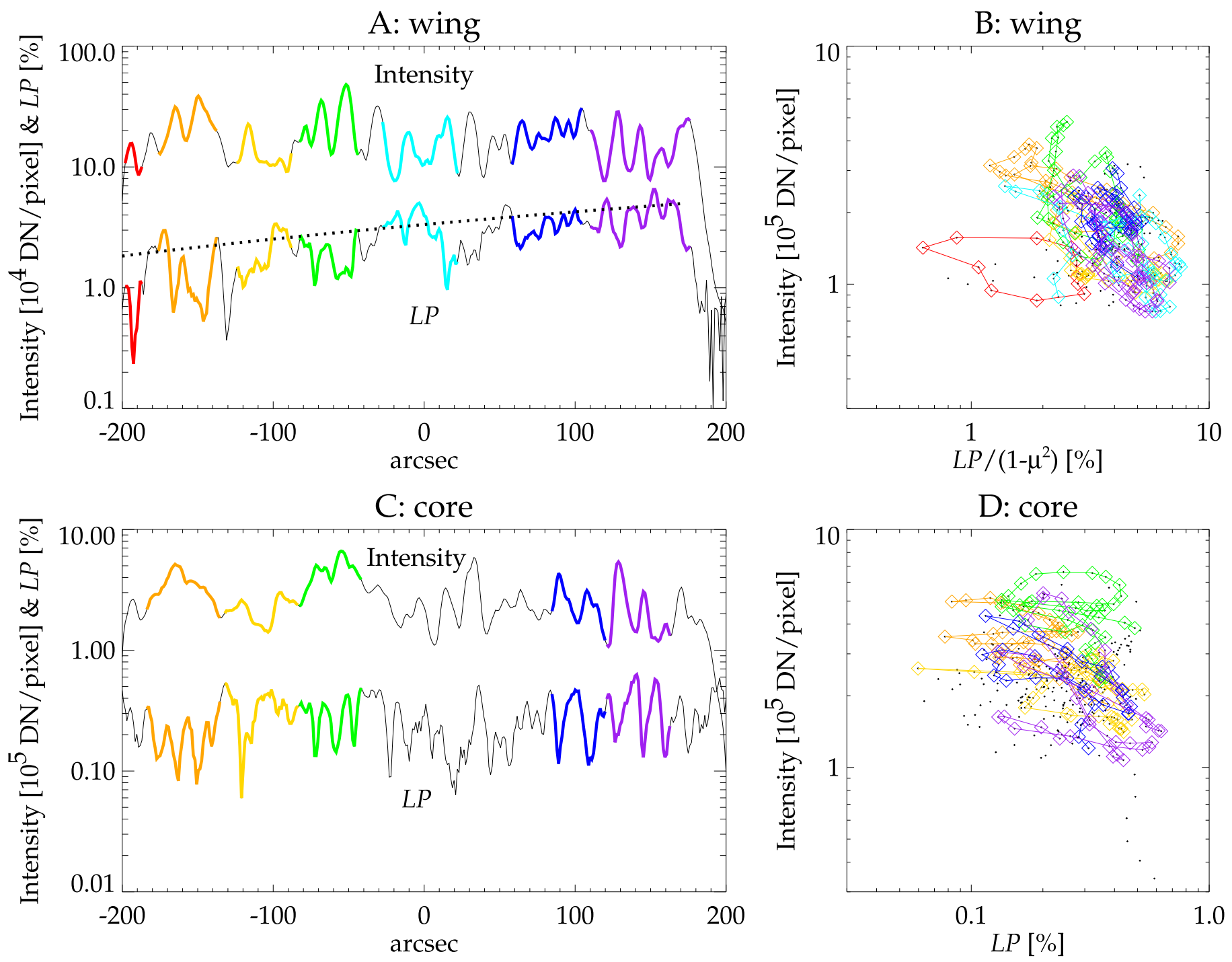

Figure 4. Spatial correlation between the intensity and the linear polarization degree LP. (A) The spatial variation of the intensity (upper curve) and LP (lower curve) at the Ly $\alpha$ wing. The segments having a clear anticorrelation between them are indicated by colors. (B) Scatter plot between LP and the intensity at the Ly $\alpha$ wing. The same colors are used for the data points in the colored segments. (C) and (D) are the same for the Ly $\alpha$ core. Only in panel (B) is LP divided by (1 - $\left.\mu^{2}\right)$ to remove the CLV trend.

(10-100 Gauss) magnetic fields in the enigmatic TR. The lack of CLV in $Q / I$ at line center is, however, an important and intriguing surprise, whose theoretical interpretation must significantly alter our empirical understanding of the solar TR. This is because the significant CLV at the center of the Ly $\alpha Q / I$ profile predicted by state-of-the-art 3D models of the solar atmosphere (see Štěpán et al. 2015) can be made to disappear either by increasing the magnetization and/or the geometrical complexity of the model's chromosphere-corona TR, which is intimately related to the magnetic-field topology (J. Štěpán et al. 2017, in preparation; J. Trujillo Bueno et al. 2017 , in preparation).

Finally, the non-trivial fact that the upper chromosphere and TR of the Sun generate significant linear polarization signals in the (optically thick) hydrogen $\operatorname{Ly} \alpha$ line of the solar disk radiation supports the theoretical expectation that other ultraviolet lines with complementary magnetic sensitivities, such as the He II line at $30.4 \mathrm{~nm}$ (Trujillo Bueno et al. 2012) and the $\mathrm{Mg}$ II $\mathrm{h} \& \mathrm{k}$ lines around $280.0 \mathrm{~nm}$ (Belluzzi \& Trujillo Bueno 2012), should also be linearly polarized by anisotropic radiation pumping processes and that vacuum-ultraviolet spectropolarimetry is a key gateway to the magnetism and geometry of the outer atmosphere of the Sun and other stars. To fully open up this new diagnostic window, we need highprecision instruments like CLASP on board space telescopes.

The CLASP team is an international partnership between NASA Marshall Space Flight Center, National Astronomical Observatory of Japan (NAOJ), Japan Aerospace Exploration Agency (JAXA), Instituto de Astrofísica de Canarias (IAC), and Institut d'Astrophysique Spatiale; additional partners are the Astronomical Institute ASCR, Istituto Ricerche Solari Locarno (IRSOL), Lockheed Martin, and University of Oslo. The U.S. participation was funded by NASA Low Cost Access to Space (award number 12-SHP 12/2-0283). The Japanese participation was funded by the basic research program of ISAS/JAXA, internal research funding of NAOJ, and JSPS KAKENHI grant numbers 23340052, 24740134, 24340040, and 25220703. The Spanish participation was funded by the Ministry of Economy and Competitiveness through project 
AYA2010-18029 (Solar Magnetism and Astrophysical Spectropolarimetry). The French hardware participation was funded by Centre National d'Etudes Spatiales (CNES). J.T.B. and J.S. gratefully acknowledge the supercomputing grants provided by the Barcelona Supercomputing Center (National Supercomputing Center, Barcelona, Spain).

\section{References}

Belluzzi, L., \& Trujillo Bueno, J. 2012, ApJL, 750, L11

Belluzzi, L., Trujillo Bueno, J., \& Štěpán, J. 2012, ApJL, 755, L2

De Rosa, M. L., Schrijver, C. J., Barnes, G., et al. 2009, ApJ, 696, 1780

Giono, G., Ishikawa, R., Narukage, N., et al. 2016, SoPh, 291, 3831

Giono, G., Ishikawa, R., Narukage, N., et al. 2017, SoPh, 292, 57

Hansteen, V., De Pontieu, B., Carlsson, M., et al. 2014, Sci, 346, 1255757

Ishikawa, R., Kano, R., Bando, T., et al. 2013, ApOpt, 52, 8205

Ishikawa, R., Narukage, N., Kubo, M., et al. 2014, SoPh, 289, 4727

Ishikawa, S., Shimizu, T., Kano, R., et al. 2015, SoPh, 290, 3081

Kano, R., Bando, T., Narukage, N., et al. 2012, Proc. SPIE, 8443, 84434F

Kneer, F. 2010, MmSAI, 81, 604
Kobayashi, K., Kano, R., Trujillo-Bueno, J., et al. 2012, in ASP Conf. Ser. 456 Fifth Hinode Science Meeting, ed. L. Golub, I. De Moortel, \& T. Shimizu (San Francisco, CA: ASP), 233

Kubo, M., Katsukawa, Y., Suematsu, Y., et al. 2016, ApJ, 832, 141

Landi Degl'Innocenti, E. 1998, Natur, 392, 256

Landi Degl'Innocenti, E., \& Landolfi, M. 2004, Polarization in Spectral Lines (Dordrecht: Kluwer)

Manso Sainz, R., \& Trujillo Bueno, J. 2003, PhRvL, 91, 111102

Narukage, N., Auchère, F., Ishikawa, R., et al. 2015, ApOpt, 54, 2080

Narukage, N., Kubo, M., Ishikawa, R., et al. 2017, SoPh, 292, 40

Pereira, T. M. D., De Pontieu, B., Carlsson, M., et al. 2014, ApJL, 792, L15

Priest, E. 2014, Magnetohydrodynamics of the Sun (Cambridge: Cambridge Univ. Press)

Rutten, R. J., \& Rouppe van der Voort, L. H. M. 2017, A\&A, 597, A138

Stenflo, J. O., Dravins, D., Wihlborg, N., et al. 1980, SoPh, 66, 13

Stenflo, J. O., Keller, C. U., \& Gandorfer, A. 2000, A\&A, 355, 789

Štěpán, J., Trujillo Bueno, J., Leenaarts, J., \& Carlsson, M. 2015, ApJ, 803, 65

Trujillo Bueno, J. 2014, in ASP Conf. Ser. 489 Solar Polarization 7, ed K. N. Nagendra, J. O. Stenflo, Z. Q. Qu, \& M. Sampoorna (San Francisco, CA: ASP), 137

Trujillo Bueno, J., Štěpán, J., \& Belluzzi, L. 2012, ApJL, 746, L9

Trujillo Bueno, J., Štěpán, J., \& Casini, R. 2011, ApJL, 738, L11 